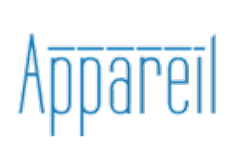

Appareil

4 | 2010

Biopolitique, éthique et subjectivation, questions de modernité

\title{
Foucault : le corps, le pouvoir, la prison
}

\section{Martine Lefeuvre Déotte}

\section{(2) OpenEdition \\ Journals}

Édition électronique

URL : https://journals.openedition.org/appareil/901

DOI : 10.4000/appareil.901

ISSN : 2101-0714

Éditeur

MSH Paris Nord

Référence électronique

Martine Lefeuvre Déotte, «Foucault : le corps, le pouvoir, la prison », Appareil [En ligne], 4 | 2010, mis en ligne le 27 janvier 2010, consulté le 12 octobre 2021. URL : http://journals.openedition.org/appareil/ 901 ; DOI : https://doi.org/10.4000/appareil.901

Ce document a été généré automatiquement le 12 octobre 2021.

\section{(c)}

Appareil est mis à disposition selon les termes de la Licence Creative Commons Attribution - Pas d'Utilisation Commerciale - Pas de Modification 4.0 International. 


\title{
Foucault : le corps, le pouvoir, la prison
}

\author{
Martine Lefeuvre Déotte
}

1 Je propose de revenir sur le texte de M. Foucault, Surveiller et punir. Naissance de la prison, cherchant à analyser les rapports du pouvoir et du corps. Ayant travaillé comme enseignante dans la plus grande prison d'Europe, Fleury-Mérogis, ouverte en 1969, qui stocke en moyenne 5000 détenus pour une capacité de 3400 places, la première impression a bien été celle d'une immense cage. Fleury gère la population, organise et trie les flux et dans son immense bonté protège les corps du suicide en voilant les cages des escaliers trop vertigineux par des filets de protection. La lecture de ce texte a toujours été précieuse, même si, il faut l'avouer, lire Foucault tout en travaillant en prison relève d'une certaine forme de masochisme.

2 En 1971, Foucault organise un séminaire au Collège de France sur le «parricide aux yeux roux ", Pierre Rivière, responsable d'un triple meurtre et jugé en août 1835 à la Cour d'assises de Caen. La même année, il crée le GIP (groupe d'information sur les prisons) avec Pierre Vidal-Naquet et Jean-Marie Domenach et la presse rend compte en 1971-1972 du " grondement de la bataille ", c'est-à-dire des insurrections, qui secouent en France trente-cinq établissements pénitentiaires ; Foucault publie enfin Surveiller et punir. Naissance de la prison en 1975. Face aux institutions judiciaires et carcérales, l'engagement théorique et militant de Foucault est évident.

3 Surveiller et punir qui aurait dû s'appeler "punir » (référence à l'Ancien Régime) et « surveiller » (référence à la Modernité) est un ouvrage foisonnant, complexe, mais qui s'appuie sur une solide lecture des archives.

4 Les relations entre le corps et le pouvoir seront analysées ici, sous l'angle unique du traitement différentiel du corps des condamnés par les instances de pouvoir. La lecture est ici volontairement partielle, pour tenter de systématiser ce rapport corps/pouvoir. Les questions soulevées dans Surveiller et punir par Michel Foucault pourraient se formuler ainsi : comment les sociétés gouvernent-elles les corps et les esprits pour les rendre dociles et soumis ? Quels sont les techniques et les savoirs, les pratiques et les discours qu'elles déploient pour maintenir l'ordre? Comment le corps devient-il un 
objet pour une connaissance, « une mise en objectivité » et une prise pour un pouvoir ? Ou encore comment se lient le pouvoir et le savoir notamment dans cette généalogie de la peine ? Je dégagerai trois manières de châtier les coupables, trois finalités distinctes de la peine.

\section{Supplice du corps}

5 L'ordonnance de 1670, en France, régit jusqu'à la Révolution la pratique pénale et hiérarchise selon les délits, les divers châtiments : la mort, la question, les galères, le fouet, l'amende honorable, le bannissement, le blâme, le cachot et les amendes. Ces punitions qui ne s'attaquent pas toutes au corps du condamné, sont le plus souvent accompagnées d'une dimension de supplice, de souffrance qui doit absolument s'offrir en spectacle : pilori, carcan, fouet, marque. Les corps corrigés sont des corps frappés du sceau indélébile de l'infamie, qui les exclut à tout jamais de la société des «honnêtes gens ", d'où la violence extrême des inscriptions tégumentaires : lettres GAL gravées à même la peau et qui signalent le passage par les galères royales, voleurs désignés par la lettre $\mathrm{V}$ calligraphiée au fer rouge. La marque se déchiffre et signale les hors la loi. Ce supplément de souffrance dans la correction répond à une double exigence : bannir et manifester avec éclat le pouvoir de celui qui punit. Aux yeux de tous, la loi du souverain s'empare des corps récalcitrants et réaffirme avec force son pouvoir. Si l'établissement de la vérité des faits, de la culpabilité, est une procédure secrète même pour le condamné : «le droit de punir ne peut en aucun cas appartenir à la "multitude": devant la justice du souverain, toutes les voix doivent se taire " $(1975$, p. 40), au contraire la mise au ban, par la torture des corps, s'effectue en pleine lumière pour terroriser et enchanter la foule. Il s'agit là d'un rituel politique, au même titre que les fastes organisés pour le couronnement ou la mort du souverain, «Le supplice ne rétablissait pas la justice ; il réactivait le pouvoir» $(1975$, p. 53$)$. Le prince affirme et met en scène la dissymétrie des rapports sociaux, il se venge d'avoir été trahi par son sujet, toujours responsable d'un crimen majestatis (crime contre la majesté) et organise la mise en pièces du corps du condamné. Il invite son peuple à la grande fête punitive, susceptible à tout instant de basculer dans l'émeute. Ceux qui n'ont plus rien à perdre peuvent crier leur innocence, leur haine et leur mépris des lois. La foule risque alors d'acclamer un héros et de prendre sa revanche contre les puissants. Rituel ambigu et paradoxal qui contient une part de carnaval: les rôles peuvent à tout moment s'inverser. Le condamné peut être applaudi et le prince fustigé. Fondamentalement, écrit Foucault, « il faut concevoir le supplice, tel qu'il est ritualisé encore au XviII ${ }^{\mathrm{e}}$ siècle, comme un opérateur politique" $(1975$, p. 57). Sous l'Ancien Régime, le souverain s'empare du corps du condamné et le torture en public, les significations de cette sanction sont multiples : châtier, se venger, effrayer et manifester rituellement sa toute puissance, son exception, celle du droit de vie et de mort sur ses sujets.

\section{2. Élision du corps}

6 À la charnière du XVIII et du XIX ${ }^{e}$ siècle, on assiste à une rupture dans la généalogie de la peine ; le pouvoir, qui n'appartient plus au roi mais dans l'idéal au peuple, investit le corps différemment et transforme dans le même temps la finalité des sanctions. À la fureur du prince succède la punition impersonnelle, mais systématique et régulière de 
la société. La justice criminelle au lieu de se venger doit punir mieux et protéger la société. Le réformateur milanais, Cesare Beccaria (1738-1794), dans le sillage des Lumières, va être l'un des précurseurs de cette transformation. Il publie en 1764 son traité Des délits et des peines, qui se répandra en Europe et sera acclamé par Voltaire, Diderot et d'Alembert. Il souligne la barbarie et l'incohérence des châtiments infligés au corps du condamné. «La torture est le plus sûr moyen d'absoudre les scélérats robustes et de condamner les innocents débiles" (1991, p.96). Il plaide pour une peine rationnelle, juste et plus humaine; il dénonce la pratique de l'aveu extorqué sous les coups, la peine de mort et préconise le système de la preuve pour désigner le coupable. Beccaria est un utilitariste, un homme d'ordre, qui propose une approche objective et rationalisée du crime :

Le but des châtiments ne peut être dès lors que d'empêcher le coupable de causer de nouveaux dommages à ses concitoyens et de dissuader les autres d'en commettre de semblables. Il faut donc choisir des peines qui, toute proportion gardée, fassent l'impression la plus efficace et la plus durable possible sur l'esprit des hommes, et la moins cruelle sur le corps du coupable (édition de 1991, p. 87).

7 S'opère un glissement dans cette volonté d'adoucissement des peines; ces dernières plus humaines et plus rationnelles doivent frapper non plus les corps mais les esprits. La punition ne doit plus bannir et stigmatiser, elle accède à un autre rôle, elle doit amender les individus et défendre la société. Le corps s'efface en partie pour ne devenir dans l'esprit des réformateurs, qu'un simple intermédiaire, un instrument dans cette entreprise de rééducation des «âmes». La pénalité se veut incorporelle, le châtiment doit s'attaquer au cœur, à la pensée, à la volonté, aux dispositions, plutôt qu'au corps. Il convient d'amplifier non pas la souffrance mais la représentation du châtiment « la "peine" de la peine ». Les hommes honnêtes doivent être épouvantés par l'idée même de la punition, seule capable de dissuader.

Le frein le plus puissant pour arrêter les crimes n'est pas le spectacle terrible mais momentané de la mort d'un scélérat, c'est le tourment d'un homme privé de sa liberté, transformé en bête de somme et qui paie par ses fatigues le tort qu'il a fait à la société (Beccaria 1991, p. 128).

Cette pénalité qui s'attaque aux esprits, prend la forme chez les réformateurs des travaux publics par exemple; elle doit dans l'idéal prévenir les infractions, assurer l'ordre, corriger l'individu, pour le réintégrer dans la société. Pour reprendre les analyses de Norbert Elias, avec le processus de civilisation, la pacification des mœurs, le rapport à la violence s'est transformé, le spectacle de la mise en pièces devient insupportable.

Élision du corps, contrôle et adoucissement des punitions, pédagogie du châtiment, protection de la société, là sont les idéaux des réformateurs.

\section{Enfermement, surveillance et discipline du corps}

En France, mais aussi en Angleterre, aux USA, la détention, la privation de la liberté, devient finalement la forme essentielle du châtiment dès le Code pénal de 1810. Le mot prison, en français, vient du latin prensionem, qui marque l'action d'arrêter. La prison, c'est à la fois la prise des corps, leur emprisonnement, puis l'endroit où on les enferme. On assiste à la multiplication des prisons au xix siècle et au quasi-abandon de la diversité des autres punitions. Les corps détenus sont alors investis différemment par les rapports de pouvoir, ils deviennent avant tout des objets de connaissance. Dominer 
efficacement, atteindre les individus dans leurs gestes, leurs attitudes, leurs discours, c'est dans le même mouvement, développer des connaissances rigoureuses permettant le dressage: la psychologie, la psychiatrie, la criminologie, la médecine seront complices de ce contrôle. Le pouvoir, loin d'empêcher le savoir, le produit. Intrication chez Foucault du pouvoir et du savoir. Accumuler sur chacun une archive, noter les comportements, questionner: toutes ces petites techniques de notation permettent l'émergence des sciences de l'homme. S'ouvre au XIXe siècle, l'époque des "disciplines ", aux deux sens du terme : sorte de fouet fait de cordelettes ou de petites chaînes utilisées pour se mortifier, se flageller, mais aussi constitution d'un ensemble de savoirs. Le $\mathrm{XIX}^{\mathrm{e}}$ siècle voit l'expansion des institutions disciplinaires: armées, hôpitaux, écoles, asiles, ateliers; elles mettent au point des procédures pour contrôler, mesurer, dresser et surveiller les individus afin de les rendre fondamentalement dociles et utiles. Dans ces lieux clos, «total institutions » ainsi que l'avait déjà noté, dès 1961, le sociologue américain E. Goffman (Asylums), l'utilisation d'un emploi du temps, des exercices, manœuvres, notations, examens, classements, assurent l'assujettissement des corps, leur humiliation, la gestion des individus.

11 De la même façon, établir une peine juste, individuelle, rationnelle, qui prend en compte la personnalité du condamné en se prononçant «scientifiquement » sur son degré de responsabilité ou de "folie» au moment des faits, demande l'exercice de savoirs nouveaux capables d'apprécier la normalité et son contraire : la criminologie, la psychiatrie, la psychologie sont alors convoquées pour répondre à ces exigences et moduler les peines dans les cours de justice. L'analyse présentée par Foucault du « cas » Rivière est sur ce point éclairante. Sous l'apparence physique de l'idiot, la question est bien de savoir si Rivière est sain d'esprit. Est-il responsable de ses actes, mérite-t-il la peine de mort, les circonstances atténuantes, quelle est la juste peine? Plus que le crime, c'est le criminel qu'il convient de juger : le jugement sur le condamné implique dès lors un jugement sur la normalité et nécessite le recours à la psychiatrie et à la psychologie. Le parricide doit également passer aux aveux en expliquant par l'écriture son acte.

12 L'institution carcérale se dote de spécialistes (éducateurs, enseignants, surveillants, aumôniers, médecins) qui cherchent à étudier "l'âme» du condamné. Les corps enfermés ne sont plus, selon la loi, suppliciés mais surveillés, observés, pour mieux assujettir les esprits. Le détenu est décrit, jaugé, mesuré, comparé, et l'institution cherche à redresser son âme, qui doit devenir « la prison du corps ». Dans cette finalité des institutions disciplinaires à dresser les corps et les esprits, on retrouve une proximité dans l'analyse entre Foucault et Bourdieu avec ses concepts d'hexis corporelle, d'habitus et de violence symbolique.

On le sait, Foucault accorde au texte de Jeremy Bentham (1748-1832) Panopticon works (1791) une grande importance conceptuelle. Cette architecture utopique, le panoptique (néologisme crée par Bentham, du grec pan = tout) est donc un dispositif qui permet à un seul de tout voir. Le geôlier, installé dans une tour centrale, surveille les cellules distribuées autour de lui en anneau, sans être vu. Bentham a écrit son texte chez son frère Samuel, qui dirigeait une manufacture en Russie méridionale. Les ouvriers qualifiés venaient d'Angleterre, mais ils n'étaient pas suffisamment dociles. Pour les surveiller et les discipliner aux moindres frais, Samuel imagina une architecture efficace pour loger et surveiller rapidement 2000 ouvriers. Jérémy Bentham est séduit par la simplicité et l'efficacité du plan; pendant 20 ans, il cherchera à améliorer le 
dispositif qui pourra être utilisé dans tous les lieux nécessitant la surveillance sans relâche de plusieurs individus par un petit nombre. Il se ruinera pour acheter un terrain où il souhaitait retenir et punir efficacement 1000 prisonniers. Il avait également prévu une machine cylindrique à châtier "scientifiquement" les récalcitrants. Bentham imagine sa prison où l'inspecteur voit d'un seul coup d'œil, tout ce qui se passe, mais elle doit rester largement ouverte sur le monde : les curieux, les voyageurs, les amis des prisonniers, peuvent facilement y entrer. Les témoins extérieurs viennent régulièrement surveiller l'inspecteur de la tour centrale qui doit rendre des comptes au « grand comité public du tribunal du monde ». La prison pensée par Bentham, n'est pas un lieu secret, sans extériorité, elle doit rester publique, visible, pour prévenir tout dérèglement et tout arbitraire. À mon sens, Foucault ne nous livre qu'une lecture partiale de ce texte de Bentham, gommant ce contre-pouvoir, fondamental chez Tocqueville, celui de l'opinion publique, qui à chaque instant peut faire irruption pour juger le travail du geôlier.

Pour Foucault, cette "cage savante et cruelle » sans extériorité devient l'emblème de cette nouvelle forme de pouvoir qui se met en place au XIx ${ }^{e}$ siècle dans les institutions disciplinaires, celui de la surveillance généralisée des corps et de leur connaissance approfondie.

Le Panoptique devient la figure architecturale de cette technique de pouvoir qui trie les corps, les isole et par un effet de transparence et de dissymétrie des regards, les discipline. Pour Foucault, les institutions, c'est-à-dire l'hôpital, la manufacture, l'école, la prison, deviennent d'immenses machines à connaître, surveiller et modeler les individus. La prison panoptique, ou plus largement la société disciplinaire, jette son filet sur l'ensemble des acteurs sociaux, surtout lorsque ceux-ci présentent une anormalité quelconque : le fou, le délinquant, le criminel. Il convient de trier le bon grain de l'ivraie, d'exclure certains pour protéger les autres : assurer la sécurité et la protection de la population, caractérise, pour Foucault, la figure du pouvoir moderne, le biopouvoir.

Face à cet implacable pouvoir d'assujettissement, sans extériorité, la «déprise » des corps est-elle même envisageable? Le pouvoir n'est-il qu'une entière et totale coercition comme souvent chez Bourdieu ? On retrouve là le vieux débat sociologique entre holisme et individualisme.

Foucault n'achève pas son livre, l'analyse théorique est comme suspendue par les luttes dans les prisons. Le « tout carcéral » permettrait-il alors la rébellion? À Metz comme à Nancy, ce sont des corps qui refusent, au quotidien, le «mitard» (cette prison plus féroce encore à l'intérieur de la prison), le parloir (espace où l'on reçoit les visites) et ses exigences, les coups, les sévices, l'obligation du port de l'uniforme, la privation de nourriture, la censure des journaux, le tutoiement systématique etc. Ce sont également des esprits qui, par le biais de l'écriture, analysent leur délit et dénoncent les inégalités sociales. Le GIP (Groupe d'information sur les prisons) souhaitait vivement ouvrir à la prise de parole ceux qui en étaient totalement privés, l'information à l'état brut devenait une lutte. Les familles, les détenus devaient décrire par le menu la violence inadmissible infligée aux corps par l'institution, les petits détails étaient essentiels : les détenus sont enchaînés au mitard de Toul, attachés dans des cachots avec une camisole de force à la Santé, disposent seulement d'un seau pour WC à Nevers etc. La publicité la plus grande devait être accordée à toutes ces atteintes aux droits fondamentaux, pour 
que l'extérieur prenne conscience de la part «d'intolérable » qui régnait dans les prisons françaises.

Il fallait faire entrer la prison dans l'actualité, non sous forme de problème moral, ou de gestion générale, mais comme un lieu où il se passe de l'histoire, du quotidien, de la vie, des évènements, du même ordre qu'une grève dans un atelier, un mouvement de revendication dans un quartier, une protestation dans une cité HLM (Foucault, 1994, p. 86).

Il convenait absolument de donner la parole à ceux qui en étaient privés pour qu'ils formulent les sévices dont ils étaient victimes.

L'expérience de la division, prend ici la figure singulière d'une mobilisation des corps, d'une révolte des corps contre l'inacceptable, elle s'inscrit à l'intérieur d'une histoire immédiate, qui ne désigne en rien un ennemi principal, ne propose pas un programme politique, n'attend pas des lendemains qui chantent. Les détenus cherchent essentiellement à défendre leurs besoins fondamentaux, leurs droits à la vie. Témoigner, c'est lutter et la révolte des prisons apportera effectivement une série de réformes.

Quel rapide bilan dresser aujourd'hui sur les prisons françaises qui ne cessent de se multiplier et de tourner à plein régime : on y stocke, dans la confusion, tous ceux qui doivent purger leur peine, mais aussi la masse des toxicomanes, des «fous", des sanspapiers, déclarés ennemis, maudits donc légalement sacrifiables. Finalement, pour reprendre l'analyse de L. Wacquant sur les prisons aux USA, on parque la misère, on l'isole, on la stigmatise, on la laisse mourir : dépérissement de l'État social et prospérité de l'État pénal. Pour assurer la sécurité des nantis, gagner une certaine forme de paix sociale, on décrète l'abandon des plus défavorisés.

\section{BIBLIOGRAPHIE}

Beccaria Cesare, Des délits et des peines [1764], Paris, Flammarion, 1991.

Brossat Alain, Pour en finir avec la prison, Paris, La Fabrique, 2001

Foucault Michel, Surveiller et punir. Naissance de la prison, Paris, Gallimard, 1975.

Foucault Michel, Dits et écrits 1976-1979, Paris, Gallimard, 1994.

Wacquant Loïc, Les prisons de la misère, Paris, Seuil, Raisons d'agir, 1999.

\section{INDEX}

Mots-clés : prison, corps 


\section{AUTEURS}

\section{MARTINE LEFEUVRE DÉOTTE}

Maître de conférences, département de sociologie, université de Caen. CMH, DYRESO. 